\title{
ESTUDO PILOTO: APLICAÇÃO DE INFORMATIVOS NUTRICIONAIS EM SERVIÇOS DE ALIMENTAÇÃO EM PERÍODO PANDÊMICO
}

\author{
Patrícia Arruda Scheffer ${ }^{1}$; Cátia Regina Storck ${ }^{2}$; Ana Lúcia de Freitas Saccol ${ }^{3}$
}

\section{RESUMO}

O presente estudo possui como objetivo avaliar cardápios nutricionais distribuídos por meio de delivery em serviços de alimentação no período pandêmico. A pesquisa foi realizada durante o mês de agosto de 2021, na cidade de Santa Maria-RS. Os cardápios nutricionais continham três informativos nutricionais (Semáforo; Alerta; Simplificado) e foram enviados de maneira impressa aos participantes através do delivery de um restaurante comercial e uma pizzaria. A maioria dos participantes eram do sexo femino e com ensino superior completo. Os participantes responderam que o informativo nutricional Semáforo é que o mais possui influência, aceitabilidade e preferência para aplicação em cardápios. Pesquisas como esta são fundamentais para encontrar estratégias adequadas para informar os consumidores sobre os alimentos distribuídos em serviços de alimentação.

Palavras-chave: Alimentação Coletiva; Nutrição; Rotulagem Frontal.

Eixo Temático: Atenção Integral e Promoção à Saúde - AIPS

\section{INTRODUÇÃO}

Com a crescente urbanização, crescimento populacional e inserção da mulher no mercado de trabalho, cada vez mais as famílias optam por realizar suas refeições fora de casa. No Brasil, sabe-se que a procura por serviços de alimentação aumentou exponencialmente, já que em 2012 o consumo total foi de 10,9 milhões de refeições/ dia, e em um salto temporal para 2019 o consumo totalizou uma soma de

\footnotetext{
${ }^{1}$ Nutricionista. Mestranda em Ciências da Saúde e da Vida- Universidade Franciscana -UFN. Grupo de Pesquisa em Segurança Alimentar e Nutricional (GESAN/CNPq).

e-mail: patricia.scheffer@ufn.edu.br

${ }^{2}$ Docente no Curso de Nutrição- Universidade Franciscana - UFN.Grupo de Pesquisa em Segurança Alimentar e Nutricional (GESAN/CNPq). e-mail:"catiars@ufn.edu.br

${ }^{3}$ Docente no Curso de Nutrição e Mestrado em Ciências da Saúde e da Vida- Universidade Franciscana- UFN. Grupo de Pesquisa em Segurança Alimentar e Nutricional (GESAN/CNPq). e-mail: alsaccol@ufn.edu.br
} 
14,2 milhões de refeições/ dia no Brasil (ASSOCIAÇÃO BRASILEIRA DE EMPRESAS DE REFEIÇÕES COLETIVAS, 2020).

Os serviços de alimentação são locais onde ocorre a manipulação, preparação, fracionamento, armazenamento, distribuição, transporte, exposição à venda e entrega de alimentos preparados ao consumo (BRASIL, 2004). Estes locais podem ser cantinas, bufês, confeitarias, cozinhas industriais, lanchonetes, padarias, pastelarias, restaurantes, cozinhas de instituições de longa permanência, dentre outros (BRASIL, 2004;RIO GRANDE DO SUL 2009).

Em 2020, devido a pandemia da Covid-19 adaptações a uma nova realidade foram necessárias (WANG et al., 2020). Em virtude do ocorrido, medidas precisaram ser adotadas e dentre tantas restrições, os serviços de alimentação foram um dos locais mais afetados, e muitos tiveram de inovar para não fechar suas portas.

Com isto, a distribuição de alimentos está passando por mudanças significativas durante a pandemia de COVID-19 e a expansão das entregas de alimentos é cada vez mais visível (HOBBS,2020). Por isso, os serviços de alimentação tiveram de adotar o delivery ou serviço de pegue e leve como uma solução imediata para que a população pudesse comprar suas refeições mesmo com estes estabelecimentos fechados.

Por diversos motivos os consumidores buscam cada vez mais informações sobre os alimentos que estão comprando nos serviços de alimentação. Alergias e intolerâncias alimentares, presença de Doenças Crônicas Não Transmissíveis (DCNT), assim como a busca por um estilo de vida mais saudável, estão associados à procura pelas informações nutricionais.

$\mathrm{O}$ aumento progressivo dos dados de obesidade elevaram-se mundialmente (MYERS; BROYLES, 2020). Não somente durante a pandemia, a obesidade é um problema de saúde pública e vem triplicando desde a década de 70 ( WHO,2020).

Desta forma, estratégias de elaboração e execução de ações de políticas públicas fazem-se necessárias, principalmente para os indivíduos que realizam as suas refeições fora do lar (CAVALCANTE et al., 2017). Por este motivo, pensando na 
promoção da saúde e na importância de proporcionar informações aos consumidores, o presente estudo possui como objetivo avaliar cardápios nutricionais distribuídos por meio de delivery em serviços de alimentação no período pandêmico.

\section{METODOLOGIA}

Este trabalho faz parte de um grande projeto denominado " Desenvolvimento de Informativos Nutricionais para Serviços de Alimentação". O projeto possui aplicação a nível estadual no Rio Grande do Sul e engloba diversas instituições de ensino, tanto públicas, quanto privadas.

Em razão da pandemia do Covid-19, a proposta inicial do projeto era a realização da testagem dos informativos nutricionais em preparações expostas no bufê, com isto a aplicação seria de maneira presencial e durante os horários de maiores movimentos nos restaurantes comerciais (almoço e jantar). Em virtude de um piloto realizado em maio, percebeu-se que não seria possível manter esta estratégia e por isso optou-se por reajustar a metodologia proposta. A equipe de pesquisa traçou novas estratégias de abordagem ao consumidor quanto aos informativos nutricionais e em agosto de 2021, um novo piloto do projeto foi desenvolvido na cidade de coordenação do projeto (Santa Maria-RS). Neste piloto, adaptações metodológicas foram realizadas em virtude do período pandêmico, levando em consideração os objetivos propostos pelo estudo.

Dentre as estratégias, optou-se pela testagem dos três informativos em um Cardápio Nutricional (figura 1). O Informativo Nutricional Semáforo é o mais completo, pois possui: nome da preparação, benefícios, informações complementares, alertas à saúde, lista de ingredientes, gramagens, medida caseira e valor energético por porção. O Informativo Nutricional Alerta possui alguns itens do Semáforo, tais como: nome da preparação, ingredientes, gramagens e valor energético; seu diferencial é a presença de uma lupa, indicando qual nutriente está alto na preparação (exemplo: sódio; gordura saturada). O terceiro informativo, 
EDUCAÇÃO, SAÚDE

ETECNOLOGIA

26 A 28 DE OUTUBRO DE 2021

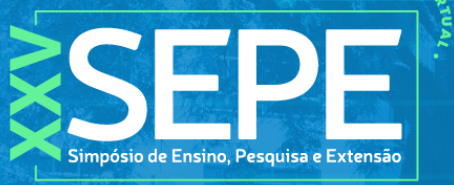

QSUFN

denominado Simplificado, possui apenas nome da preparação, ingredientes, valor calórico e medida caseira da preparação.

Figura 1- Cardápio Nutricional elaborado pela equipe do projeto, Santa Maria, RS, 2021.
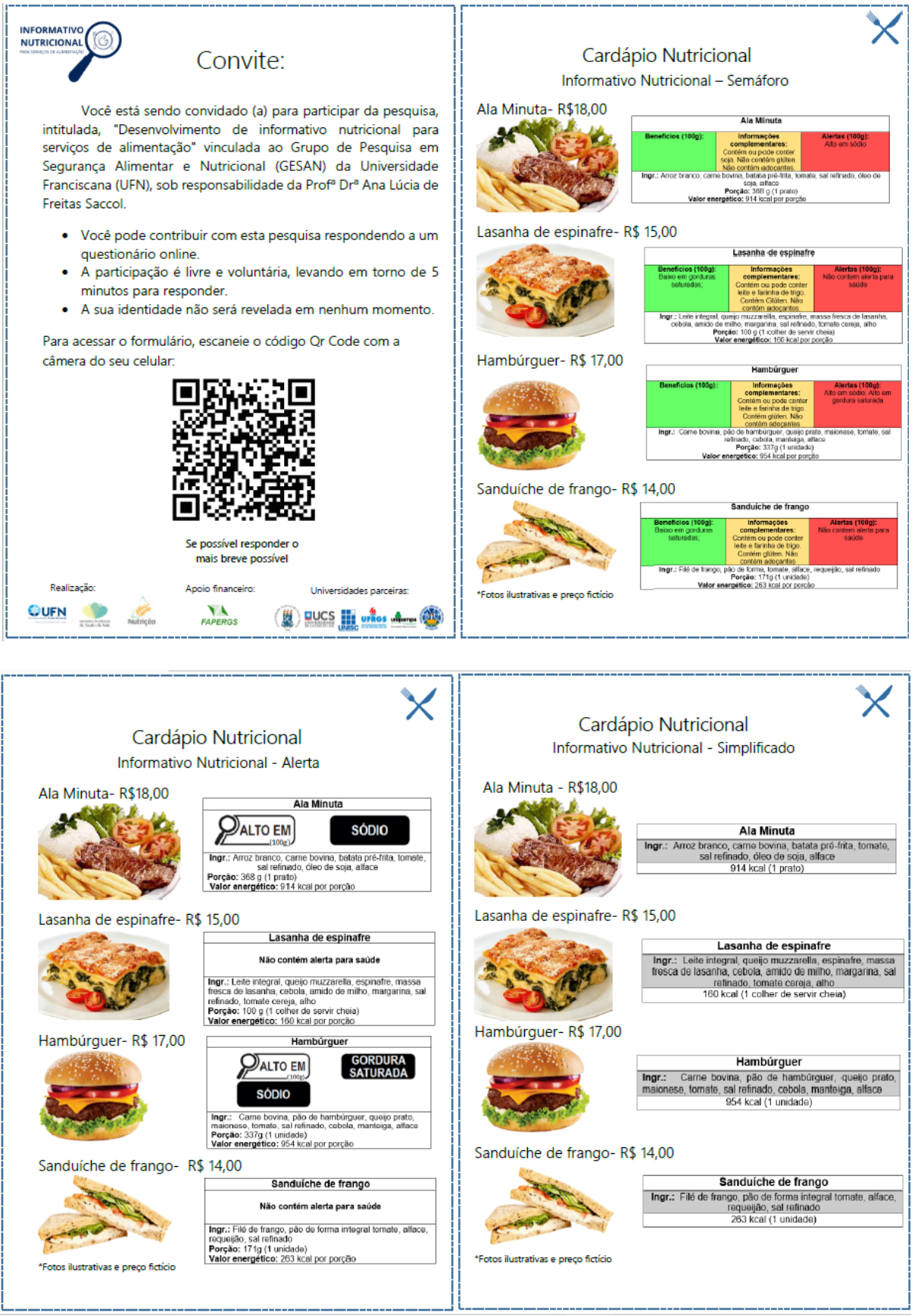
O cardápio continha quatro preparações (Ala Minuta; Lasanha de Espinafre; Hambúrguer e Sanduíche de Frango), as mesmas foram selecionadas de maneira aleatória, levando em conta que as imagens eram ilustrativas e os preços fictícios. Cada página do cardápio possuía o informativo nutricional correspondente a avaliação que o consumidor deveria realizar. Ainda, havia uma página inicial no Cardápio Nutricional, com uma breve explicação do projeto e um código QR Code, por onde os participantes possuíam acesso ao questionário da pesquisa.

O questionário era composto por questões de múltipla escolha e descritivas, dividido em dados sociodemográficos e perguntas específicas quanto aos informativos. Questões referentes às condições de saúde ( alergias, intolerâncias alimentares, alimentação saudável), também estavam incluídas no formulário enviado aos participantes.

O piloto ocorreu em dois serviços de alimentação do município, sendo um restaurante comercial e uma pizzaria. Os cardápios foram impressos e entregues 50 envelopes para cada serviço de alimentação, onde os proprietários/gerentes fizeram o envio pelo serviço de delivery ou pegue e leve para seus clientes.

Ressalta-se que, ao acessar o questionário disponibilizado por esta pesquisa, os participantes primeiro tinham acesso ao Termo de Consentimento Livre e Esclarecido (TCLE). A pesquisa possui aprovação no Comitê de Ética em Pesquisa da Universidade Franciscana (UFN), por meio do parecer 1.877.129.

\section{RESULTADOS E DISCUSSÕES}

Em um total de 100 envelopes enviados, pelos dois serviços de alimentação participantes deste estudo, obteve-se apenas $20 \%$ de participação. A maioria dos respondentes eram do sexo feminino $(66,7 \%)$ e com escolaridade com ensino superior incompleto/ completo $(57,1 \%)$. É comum encontrar em outras pesquisas a alta participação do público feminino (LASSEN et al., 2014; PÉREZ et al., 2017; SONNENBERG et al., 2013). Christoph e colaboradores (2017) acreditam que este 
fato deve-se às mulheres estarem mais preocupadas com as suas escolhas alimentares e consequentemente com sua saúde do que os homens.

Em 2021, os serviços de alimentação tiveram que se adaptar ao novo cenário que o mundo encontrava-se e com isto os serviços de entrega de refeições online aumentaram neste setor (HONG et al., 2021). Embora antes da pandemia o consumidor já adotasse o método de delivery para consumo de alimentos, de acordo com a empresa de pesquisa de varejos NPD Groups, em 2020 os serviços de entrega de alimentos aumentaram cerca de $67 \%$ comparado com os anos anteriores (NPD, 2020).

Levando em conta este aumento da procura por este tipo de serviço em restaurantes, esta estratégia foi escolhida como a principal fonte de contato com os consumidores para o envio da pesquisa. Esperava-se uma maior adesão por parte dos consumidores na pesquisa via delivery.

Diversos fatores podem estar associados à adesão dos participantes à pesquisa. Um deles é o fato da exaustão e cansaço mental da população em decorrência da pandemia, diversos estudos constataram que as pessoas estão mais tristes, menos propensas a ter disposição e entusiasmo para realizar as atividades do cotidiano (CAMACHO-ZUÑIGA et al., 2021; YARRINGTON et al.,2021). Isto pode ter influenciado, assim como o envio exacerbado de pesquisas durante este período, saturando assim a disponibilidade dos entrevistados.

Outro fator que deve ser discutido é de que, a mesma proposta de informativos nutricionais em serviços de alimentação já é estudada em outros países, em outras culturas e costumes, mas sempre com estudantes universitário (CHRISTOPH, ELLISON, 2017; GOMEZ; LE MINOUS, 2012; SEWARD; BLOCK; CHATTERJEE, 2016; WHITE et al., 2016). A maioria das pesquisas são realizadas dentro de ambientes universitários, pois geralmente este público já está acostumado a responder pesquisas científicas e compreende sua importância para novas descobertas e achados para a comunidade. 
Quando questionados quanto aos fatores que poderiam influenciar sua escolha em relação às preparações apresentadas no cardápio nutricional, nove participantes $(42,5 \%)$, responderam que a preferência pelo sabor é o principal fator a ser levado em consideração no momento da sua compra. Em contrapartida, no estudo de Sonnebeerg et al (2013), cerca de 61\% dos entrevistados responderam que a qualidade nutricional era o fator que mais influenciou suas escolhas por alimentos.

Em relação ao nível de aceitabilidade, os consumidores gostaram mais do informativo Semáforo $(57,1 \%)$. Outros estudos, associam esta aceitabilidade, devido ao fato que cada vez mais a população está em busca de informações, para que desta forma, possa modificar seus hábitos e realizar escolhas mais conscientes (POHMEIER et al., 2012; SONNEBEERG, 2013; OR et al., 2018), corroborando com os dados discutidos anteriormente.

Quando questionados qual o informativo preferido, o Semáforo ficou em primeiro lugar $(60 \%)$, e o menos preferido foi o Simplificado (40\%). Assim como no Brasil, a Bélgica realizou um estudo semelhante, onde os autores avaliaram três informativos, com características próximas aos utilizados neste estudo e concluíram que os seus participantes preferiram o informativo mais completo, pois sua população está cada vez mais preocupada com o que está consumindo (LIU; HOEFKENS; VERBEKE, 2015). Isto deve-se ao fato que, apesar do informativo Simplificado já possuir informações básicas, como preço, calorias e ingredientes, a população quer saber mais, para realizar escolhas melhores no momento da compra da sua refeição.

\section{CONCLUSÃO}

Apesar da baixa adesão por parte dos participantes, o piloto apresentou resultados interessantes e que podem corroborar para novos estudos. Pode-se concluir que em virtude do atual cenário, adaptações metodológicas serão necessárias aos projetos que foram elaborados e planejados antes deste período. 


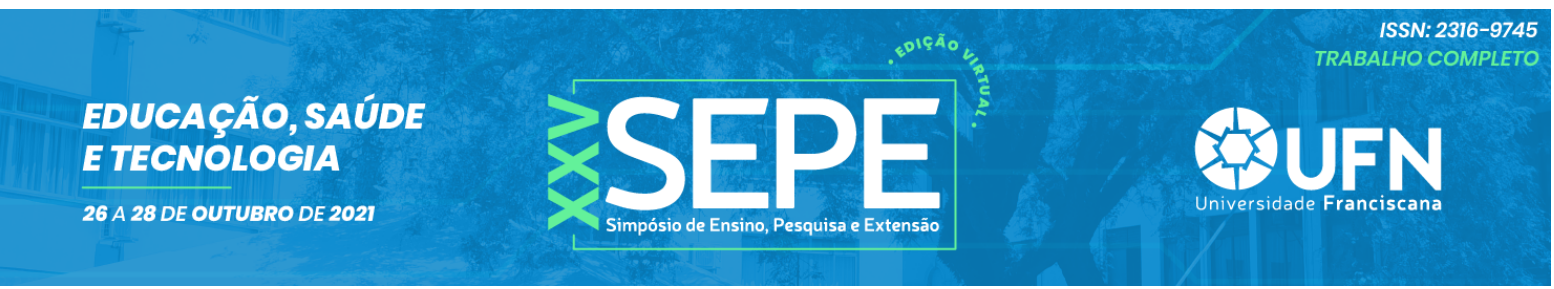

No piloto deste estudo, desejava-se alcançar a população em geral, sem restrições de níveis de escolaridade, sexo, profissão e conhecimentos técnicos sobre o tema estudado. Entende-se que a comunidade em geral será contemplada com a implementação dos informativos nutricionais nos serviços de alimentação e por isto, deve ser a população foco deste estudo.

Em razão disto, novas estratégias serão traçadas para atender aos objetivos propostos por esta pesquisa, levando em consideração o período pandêmico o qual o mundo encontra-se no momento. Pretende-se também, que a aplicação a nível estadual obtenha grande repercussão e possa ser alcançada também a nível nacional.

\section{AGRADECIMENTOS}

Fundação de Amparo à Pesquisa do Estado do Rio Grande do Sul (FAPERGS) pelo apoio financeiro neste projeto.

\section{REFERÊNCIAS}

ASSOCIAÇÃO BRASILEIRA DAS EMPRESAS DE ALIMENTAÇÃO COLETIVA. Disponivel em:https://www.aberc.com.br/mercadoreal.asp?IDMenu=21\&gt; Acesso em: 21 de set. de 2020.

BRASIL. Ministério da Saúde. Agência Nacional de Vigilância Sanitária. Resolução Diretoria Colegiada- RDC № 216, de 15 de setembro de 2004. Dispõe sobre Regulamento Técnico de Boas Práticas para Serviços de Alimentação. Dispõe sobre Regulamento Técnico de Boas Práticas para Serviços de Alimentação. 2004. Disponível em: <http://eprints.uanl.mx/5481/1/1020149995.PDF>

CAMACHO-ZUÑIGA, C. et al. The impact of the COVID-19 pandemic on students' feelings at high school, undergraduate, and postgraduate levels. Heliyon, v. 7, n. 3, p. e06465, 1 mar. 2021.

CAVALCANTE, J. B. et al. Ingestão de energia e nutrientes segundo consumo de alimentos fora do lar na Região Nordeste: Uma análise do Inquérito Nacional de Alimentação 2008-2009. Revista Brasileira de Epidemiologia, v. 20, n. 1, p. 115-123, 2017. 
CHRISTOPH, M. J.; ELLISON, B. A Cross-Sectional Study of the Relationship between Nutrition Label Use and Food Selection, Servings, and Consumption in a University Dining Setting. Journal of the Academy of Nutrition and Dietetics, v. 117 , n. 10 , p. $1528-1537,1$ out. 2017.

GOMEZ, P.; LE MINOUS, A. E. L'influence du format de l'étiquetage sur l'utilisation et la compréhension de l'information nutritionnelle: Résultats d'une expérimentation menée en restauration collective. Revue d'Epidemiologie et de Sante Publique, v. 60, n. 1, p. 9-18, 2012.

HOBBS, J. E. Food supply chains during the COVID-19 pandemic. Canadian Journal of Agricultural Economics/Revue canadienne d'agroeconomie, v. 68, n. 2, p. 171-176, 1 jun. 2020.

HONG, C. et al. Factors affecting customer intention to use online food delivery services before and during the COVID-19 pandemic. Journal of Hospitality and Tourism Management, v. 48, p. 509-518, 1 set. 2021.

LASSEN, A. D. et al. Effectiveness of offering healthy labelled meals in improving the nutritional quality of lunch meals eaten in a worksite canteen. Appetite, v. 75, p. 128-134, 1 abr. 2014.

LIU, R.; HOEFKENS, C.; VERBEKE, W. Chinese consumers' understanding and use of a food nutrition label and their determinants. Food Quality and Preference, v. 41, p. 103-111, 2015.

MYERS, C. A.; BROYLES, S. T. Fast Food Patronage and Obesity Prevalence During the COVID-19 Pandemic: An Alternative Explanation. Obesity, v. 28, n. 10, p. 1796-1797, 1 out. 2020.

NPD GROUPS. Online food orders, delivery surge amid COVID-19 lockdown. Disponível em:

https://www.npd.com/wps/portal/npd/us/news/press-releases/2020/while-total-us-rest aurant-traffic-declines-by-22-in-march-digital-and-delivery-orders-jump-by-over-60/ Acesso em: 13 de set de 2021.

OR, W. et al. The effects of traffic light labelling versus cartoon labelling on food and beverage purchases in a children's hospital setting. Pediatric obesity, v. 13, n. 4, p. 265-268, 1 abr. 2018. 
PÉREZ, C. et al. Efeito da rotulagem de calorias na seleção do menu: um estudo preliminar em Santiago, Chile. Rev Chil Nutr, v. 44, 2017.

POHLMEIER, A. et al. Using Focus Groups to Develop a Nutrition Labeling Program within University Food Service. Family and Consumer Sciences Research Journal, v. 40, n. 4, p. 431-443, 1 jun. 2012.

RIO GRANDE DO SUL. Portaria n. 78/2009. Secretaria da Saúde do Estado do Rio Grande do Sul. Aprova a Lista de Verificação em Boas Práticas para Serviços de Alimentação, aprova Normas para Cursos de Capacitação em Boas Práticas para Serviços de Alimentação e dá outras providências, Porto Alegre, 2009.

SEWARD, M. W.; BLOCK, J. P.; CHATTERJEE, A. A traffic-light label intervention and dietary choices in college cafeterias. American Journal of Public Health, v. 106, n. 10, p. 1808-1814, 1 out. 2016.

SONNENBERG, L. et al. A traffic light food labeling intervention increases consumer awareness of health and healthy choices at the point-of-purchase. Preventive Medicine, v. 57, n. 4, p. 253-257, out. 2013.

WANG, C. et al. A novel coronavirus outbreak of global health concern. The Lancet, v. 395, n. 10223 , p. $470-473,15$ fev. 2020.

WHITE, C. M. et al. A voluntary nutrition labeling program in restaurants: Consumer awareness, use of nutrition information, and food selection. Preventive Medicine Reports, v. 4, p. 474-480, 1 dez. 2016.

WORLD HEALTH ORGANIZATION. Obesity and overweight, 2020.Disponivel em: https://www.who.int/news-room/fact-sheets/detail/obesity-and-overweight

Acesso em: 12 de março de 2021

YARRINGTON, J. S. et al. Impact of the COVID-19 Pandemic on Mental Health among 157,213 Americans. Journal of Affective Disorders, v. 286, p. 64-70, 1 maio 2021. 